\title{
Combined transplantation of skeletal myoblasts and angiopoietic progenitor cells reduces infarct size and apoptosis and improves cardiac function in chronic ischemic heart failure
}

Nikolaos Bonaros, MD, ${ }^{a}$ Rauend Rauf, MD, ${ }^{\text {a }}$ Dominik Wolf, MD, ${ }^{\mathrm{b}}$ Eva Margreiter, PhD, ${ }^{\mathrm{d}}$ Alexandar Tzankov, MD, ${ }^{\mathrm{c}}$ Bernhard Schlechta, MD, ${ }^{e}$ Alfred Kocher, MD, ${ }^{e}$ Harald Ott, MD, ${ }^{a}$ Thomas Schachner, MD, ${ }^{a}$ Steffen Hering, PhD, Johannes Bonatti, MD, and Guenther Laufer, MD ${ }^{a}$

Supplemental material is available online.
From the Departments of Cardiac Surgery, Hematology, ${ }^{\mathrm{b}}$ and Pathology, ${ }^{\mathrm{c}}$ Innsbruck Medical University, Innsbruck, Austria; Innovacell Biotechnology, ${ }^{\mathrm{d}}$ Innsbruck, Austria; the Department of Cardiothoracic Surgery, Vienna Medical University ${ }^{\mathrm{e}}$; and the Institute of Pharmacology and Toxicology, University of Vienna, ${ }^{\mathrm{f}}$ Vienna, Austria.

This study was supported by a research grant of the Tyrolean Hospital Facilities and the Federal State of Tyrol.

Received for publication March 23, 2006; accepted for publication July 7, 2006.

Address for reprints: Nikolaos Bonaros, MD, Department of Cardiac Surgery, Innsbruck Medical University, Anichstrasse 35, A-6020, Innsbruck, Austria (E-mail: nikolaos. bonaros@uibk.ac.at).

J Thorac Cardiovasc Surg 2006;132:1321-8

0022-5223/\$32.00

Copyright $\odot 2006$ by The American Association for Thoracic Surgery

doi:10.1016/j.jtcvs.2006.07.023
Objectives: Cellular cardiomyoplasty using skeletal myoblasts or angiopoietic progenitor cells offers a promising approach for the treatment of ischemic heart failure. Although several studies have shown encouraging results in acute myocardial infarction, the efficacy of cell therapy using skeletal myoblasts and angiopoietic progenitor cells in chronic ischemic heart disease remains undetermined.

Methods: Ischemic heart failure was induced by left anterior descending coronary artery ligation in nude rats: (1) Culture medium, (2) homologous skeletal myoblasts (SM), (3) human AC-133+ cells (SC), and (4) both skeletal myoblasts and AC$133+$ cells $(\mathrm{Comb})$ were injected in the infarct (SM) and peri-infarct area (SC) 4 weeks after infarction. Assessment of myocardial function included echocardiography 4 weeks after cell delivery. Histology was based on quantification of myocardial fibrosis, apoptosis, and capillary density.

Results: Left ventricular dilatation was attenuated and ejection fraction improved significantly after cell transplantation (SM: $59.4 \% \pm 8.8 \%$, SC: $60.3 \% \pm 6.6 \%$, Comb: $68.2 \% \pm 5.6 \%$ vs control: $41.5 \% \pm 7.4 \%, P=.0013$ ). Quantification of scar tissue showed a significant reduction of infarct area in cell-treated animals (SM: $22.3 \% \pm 9.1 \%$, SC: $19.8 \% \pm 7.6 \%$, Comb: $13.2 \% \pm 5.8 \%$ vs controls: $36.5 \% \pm$ $8.2 \%, P=.008)$. Improvement of myocardial function was associated with reduced apoptotic index (SM: $3.2 \% \pm 0.9 \%$, SC: $3.1 \% \pm 0.6 \%$, Comb: $1.8 \% \pm 0.8 \%$ vs controls: $10.3 \% \pm 1.6 \%, P=.0002$ ) and increased vascular density (SM: $5.2 \pm 1.2$, SC: $8.3 \pm 1.8$, Comb: $12.3 \pm 2.3$, controls: $1.9 \pm 0.3$, all capillary vessels/highpower field, $P=.007$ ) in animals after cellular cardiomyoplasty.

Conclusions: Combined transplantation of skeletal myoblasts and angiopoietic progenitor cells results in ventricular function improvement, reduction of scar size and myocardial apoptosis, and increased neoangiogenesis in chronic ischemia. Clinical studies are warranted to prove this new therapeutic concept.

$\mathrm{S}$ keletal myoblast transplantation into the ischemic heart can improve myocardial function with a passive girdling effect by limiting progressive cavity dilatation. ${ }^{1,2}$ Recent studies have provided additional information regarding the possible mechanisms and set the pace for preliminary clinical studies. ${ }^{3,4}$ Those studies have shown that skeletal myoblasts possess a substantial angiogenic potential and are able to partially replace infarcted myocardium and reverse remodeling provided that they survive in large numbers in the host. ${ }^{5-7}$

Several drawbacks have limited the initial optimism of myoblast-based myocardial regeneration. First, although engrafted skeletal myoblasts form beating struc- 


\section{Abbreviations and Acronyms \\ LVEDD = left ventricular end-diastolic diameter \\ $\mathrm{LVEF}=$ left ventricular ejection fraction \\ TUNEL $=$ deoxyuride- $5^{\prime}$-triphosphate biotin nick end labeling \\ VEGF = vascular endothelial growth factor \\ YFP = yellow fluorescent protein}

have been described. ${ }^{14}$ Stable transgenic rat skeletal myoblasts were generated by a plasmid encoding for the yellow fluorescent protein (YFP) (Invitrogen Corporation, Vienna, Austria) containing the neomycin resistance gene for subsequent selection of stable YFP+ clones. Purity of clonal myoblast cultures was proved by means of desmin immunohistology (IMMH-5, Sigma, St Louis, Mo) and antiskeletal myosin-fast antibodies (clone My-32, Sigma). All sections were overlaid to pictures obtained by fluorescence microscopy and were double-confirmed with myosin heavy chain fast-slow staining (clone My-32, Sigma) to confirm cell tracking.

tures in the infarct scar, they beat asynchronously to native cardiomyocytes. $^{8}$ In addition, survival of engrafted cell is less than $1 \%$, and the average improvement of left ventricular ejection fraction (LVEF) after myoblast transplantation does not exceed $8 \% .^{3,4,7}$ Therefore, strategies to improve viability of the peri-infarct area are required to warrant a better environment for the engrafted cells.

Angiopoietic progenitor cells have been shown to improve blood and oxygen supply in the scar. The earliest precursor of both hematopoietic and endothelial cell lineage derived from embryonic ventral endothelium expresses vascular endothelial growth factor (VEGF) receptors, as well as GATA-2, $\alpha 4$-integrins, and the AC133-receptor. ${ }^{9}$ AC-133+ cells possess paracrine properties and have been shown to induce angiogenesis by means of angiogenic factors secretion. ${ }^{10}$ Their ability to transdifferentiate into endothelial cells with high proliferation capacity is associated with new vessel formation mainly attributed to vasculogenesis. ${ }^{11} \mathrm{Al}-$ though AC-133 + cells do express early cardiac markers, ${ }^{12}$ their potential to transdifferentiate into functional cardiomyocytes has been questioned. ${ }^{13}$

In the current study we evaluated the efficacy of a combined transplantation of (1) AC-133+ progenitors for induction of angiogenesis in the still viable border zone and (2) skeletal myoblasts for replacement of lost contractile structures in the infarct scar in a rodent model of chronic ischemic heart failure.

\section{Methods \\ Model of Myocardial Infarction and Ischemic Heart Failure}

Myocardial infarction was induced in 8- to 10-week-old male rnu/rnu rats (Harlan Winkelmann, Borchen, Germany) through left anterior descending coronary artery ligation. All animals received humane care in compliance with the "Principles of Laboratory Animal Care," formulated by the National Society for Medical Research, and the "Guide for the Care and Use of Laboratory Animals," prepared by the Institute of Laboratory Animal Resource and published by the National Institutes of Health.

\section{Isolation, Labeling, and Culture of Skeletal Myoblasts From Syngeneic Rats}

Rat skeletal myoblasts were isolated from 6-week-old male F344 rats (syngeneic rats). Isolation, culture, and expansion procedures
Isolation, Purification, and Labeling of Human-derived AC-133+ Angiopoietic Progenitors

Mononuclear cells were obtained from single-donor leukapheresis products of individuals mobilized with recombinant granulocyte colony-stimulating factor $10 \mu \mathrm{g} / \mathrm{kg}$ subcutaneously (Neupogen, Amgen, Thousand Oaks, Calif) for at least 4 days. Highly purified CD133+ cells ( $>98 \%$ positive) were obtained within 48 hours after blood collection using magnetic beads coated with monoclonal antibodies against CD133 (Miltenyi Biotech, Bergisch-Gladbach, Germany). Cell purity was controlled by means of flow cytometry using antibodies against CD45 and CD133 (Becton Dickinson, Franklin Lakes, NJ). AC-133+ cells were cultured with $10 \mu \mathrm{g} / \mathrm{mL}$ lipoprotein labeled with carbocyanine membrane 1,1-dioctadecyl3,3,3,3-tetramethyl indocarbocyanine perchlorate (Cell-Tracker CM-DiI, Molecular Probes, Leiden, The Netherlands) for subsequent cell tracking. After cell viability assessment (propidium iodide staining), aliquots of $106 \mathrm{AC}-133+$ cells in approximately $200 \mu \mathrm{L}$ of culture medium (Roswell Park Memorial Institute) were prepared for intramyocardial injections. Cell tracking included DiI fluorescence detection using fluorescence microscopy and counterstaining with human-specific anti-human leukocyte antigen Class I (clone w6/32, Sigma) using immunohistochemistry.

\section{Cell Transplantation}

Four weeks after myocardial infarction and after echocardiographic verification of chronic heart failure, rats were reanesthetized and hearts were reexposed through a left redo-thoracotomy to allow access to the infarct area and the peri-infarct rim proximally and distally to the scar. Roswell Park Memorial Institute culture medium ( $\mathrm{n}=5$ ), 106 YFP-labeled, desmin-positive skeletal myohuman-derived angiopoietic progenitor cells $(\mathrm{n}=5)$, and a combination of $5 \times 105$ YFP-labeled, desmin-positive skeletal myoblasts from syngeneic rats, and $5 \times 105$ DiI-labeled AC-133+ human-derived angiopoietic progenitor cells were injected in the peri-infarct area and the scar $(\mathrm{n}=5)$. Skeletal myoblasts and $\mathrm{AC}-133+$ cells were exclusively injected in the infarct scar and the peri-infarct area, respectively.

\section{Functional Assessment by Transthoracic Echocardiography}

Transthoracic echocardiography was performed before and 2 days after myocardial infarction, and 4 weeks after cell transplantation with an Acuson Sequoia Echocardiography system (Acuson Corporation, Mountain View, Calif), with a commercially available blasts from syngeneic rats $(\mathrm{n}=5), 106$ DiI-labeled AC-133+ 
$15-\mathrm{MHz}$ linear-array intracardiac transducer system (AcuNav, Acuson Corporation).

\section{Animal Sacrifice and Preparation for Morphologic Studies}

Rats were euthanatized, hearts were harvested, and fibrous tissues were removed. After the intracardiac blood was rinsed, the hearts were divided into 3 equally thick parts representing the base, the middle, and the apex of the heart. Each of them was snap-frozen in liquid nitrogen after being embedded in optimal cutting temperature compound (Tissue-Tec OCT Compound, Miles Inc, Elkhart, Ind). From each part, 5- $\mu \mathrm{m}$ slides were prepared using cryostat. Transplanted cells were detected by confocal fluorescence microscopy. In addition, standard hematoxylin-eosin staining was performed to permit morphologic assessment.

\section{Evaluation of Myocardial Fibrosis and Quantification of Myocardial Infarction}

To assess fibrosis in the left ventricular myocardium, heart samples were cut into transverse sections and stained with Masson's Trichrome (Merck, Darmstadt, Germany). Transverse sections were randomly obtained from the 3 parts described; after staining, they were scanned and computerized for digital image analysis. Fibrotic area fraction was calculated as the sum of the whole fibrotic area divided by the total myocardial area. The peri-infarct region or border zone was defined as the region of myocardium extending at least $0.5 \mathrm{~mm}$ from the infarct scar.

\section{Evaluation of Capillary Density}

To detect capillaries in the myocardium, frozen transverse sections were randomly obtained from all 3 heart parts and stained using an anti-CD34 monoclonal antibody (clone QBEnd10; DakoCytomation, Glostrup, Denmark). Immunohistochemical visualization was performed with the EnVision kit (DakoCytomation). The number of capillaries were counted in the border zone and distal area. Capillary density was expressed as the average number of capillaries of 5 random high-power fields.

\section{Evaluation of Apoptotic Index in the Left Ventricular Myocardium}

For in situ detection of apoptosis at the single-cell level, transferasemediated deoxyuride- 5 '-triphosphate biotin nick end labeling (TUNEL) staining was performed with the In Situ Cell Death Detection Kit/Peroxidase (Roche, Indianapolis, Ind) according to the instructions of the manufacturer. Within each field, 5 randomly selected regions of infarct scar, peri-infarct rim, and rest myocardium were examined, containing at least 250 cells per region. The results were expressed as the apoptotic index representing the ratio of apoptotic cells of the total myocardial cell population.

\section{Data Analysis}

Statistical analysis was performed with the Statistical Package for the Social Sciences 11.0 for Windows (SPSS Inc, Chicago, Ill). Data are expressed as mean \pm standard deviation. Comparisons of continuous variables among animal groups were studied by 1-way analysis of variance. Longitudinal studies comparing data within each group were achieved with paired $t$ tests.

\section{Results}

Transplanted Skeletal Myoblasts and AC-133+ Angiopoietic Progenitors Survive in the Environment of Chronic Ischemia

YFP-labeled homologous skeletal myoblasts were detected in the infarct scar (Figure 1, A). My-32 antibody staining revealed stable engraftment of skeletal myoblasts in the infarct area but not in the border zone (Figure 1, B). Engrafted cells formed organized cell aggregates (myotubes) that remained morphologically isolated from the host myocardium (Figure 1,C).

In addition, DiI + cells representing injected AC-133+ cells were detected in cryosections derived from the group that received stem-cell injections. Those cells had a completely different pattern of engraftment as they formed spot-like colonies (Figure 1,D).

In the combination group, both cell types were detected in chronic ischemic myocardium presenting patterns of engraftment similar to those in the single-cell therapy groups (Figure 1,E). AC-133 + cells were not only detected in the border zone but also in the infarct scar, although they were injected in the peri-infarct rim. This finding indicates that hematopoietic progenitors not only survive for an extended period of time after transplantation in chronic ischemic myocardium but also migrate to the infarct scar.

Combined Transplantation of Skeletal Myoblasts and AC-133 + Progenitors Reverses Left Ventricular Dilatation and Improves Systolic Heart Function We assessed left ventricular end-diastolic diameter (LVEDD) as a marker of left ventricular remodeling by means of transthoracic echocardiography. LVEDD increased after infarction and was almost doubled compared with the baseline diameter in control animals (Figure 2, A). Transplantation of either skeletal myoblasts or angiopoietic progenitors resulted in approximately $20 \%$ lower levels of LVEDD compared with controls (online Figures E1 and E2). Left ventricular dilatation was significantly reduced in animals, which received both cell types, as LVEDD decreased from $0.92 \mathrm{~mm}$ in the control group to $0.60 \mathrm{~mm}$ in the combination group.

LVEF significantly decreased from more than $70 \%$ to less than $40 \%$ after induction of myocardial infarction in all animals. Akinetic areas were detectable in the anterior wall and apex. In control animals, LVEF remained less than $40 \%$ at 4 weeks after injection of culture medium (Figure 2, $B)$. A statistically significant increase of systolic left ventricular function in animals that received cell monotherapy was observed with a relative improvement of 50\% compared with the LVEF levels early after infarction. Animals subjected to combined cell therapy had a more profound increase of LVEF, reaching a relative improvement of approximately $70 \%$ compared with baseline. To assess the impact of remodeling, LVEDD and LVEF were evaluated in 

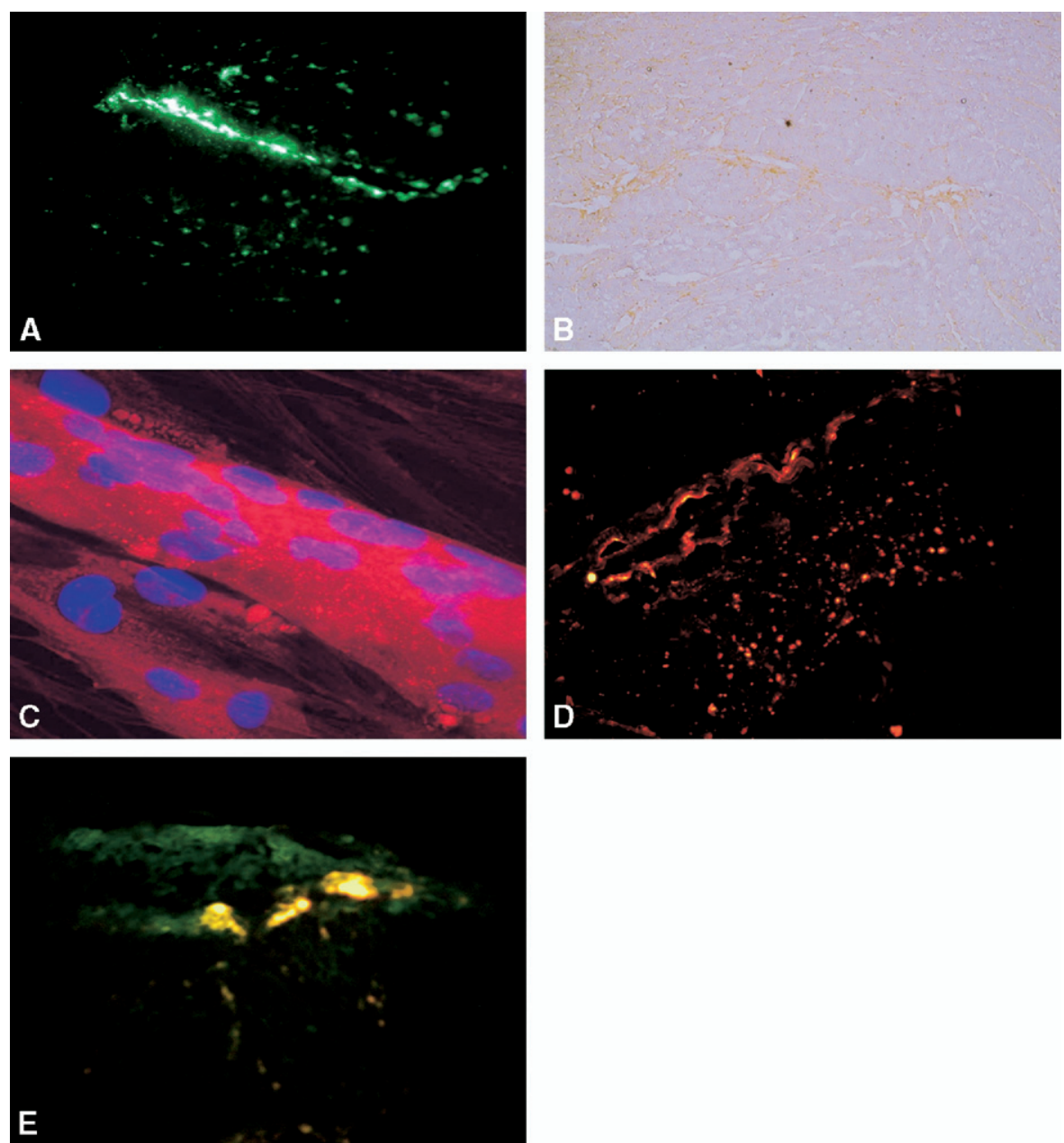

Figure 1. Transplanted skeletal myoblasts and AC-133+ angiopoietic progenitors survive in chronic ischemic myocardium. A, Fluorescent staining of YFP-labeled skeletal myoblasts (green) in the infarct area detected 4 weeks after cell injection ( $40 \times$ magnification). B, Immunohistochemical staining of skeletal myoblasts by My-32 antibody confirms fluorescence-based cell detection in the infarct scar $(40 \times$ magnification). C, Immunofluorescent staining of My-32 (red) showing stable myotube formation in the scar (cell nuclei stained blue) (100× magnification). D, Fluorescent staining of Dil-labeled AC$133+$ cells (red) in the border zone 4 weeks after cell transplantation $(40 \times$ magnification). E, Fluorescent staining of skeletal myoblasts (green) and AC$133+$ cells (red) in the infarct area 4 weeks after combined cell transplantation of skeletal myoblasts and AC$133+$ cells in the scar and border zone, respectively (100× magnification).
10 separate experiments 2 days after myocardial infarction (postoperative day 2) and 2 days before cell transplantation (postoperative day 26). No difference was observed in terms of LVEF $(39.3 \% \pm 4.2 \%$ vs $38.2 \% \pm 3.9 \%)$ between the 2 time points and before assignment to the 4 different therapy groups. LVEDD was significantly higher on postoperative day 26, suggesting postremodeling ventricular dilatation $(0.44 \pm 0.05$ vs $0.64 \pm 0.07, P=.02)$.

\section{Combined Transplantation of Skeletal Myoblasts and} Angiopoietic Progenitors Prevents Myocardial Fibrosis and Results in Reduced Myocardial Scar

We next examined whether combined cell therapy had an effect on the myocardial scar in the setting of chronic myocardial ischemia by quantifying the ratio of fibrous tissue to the left ventricle at 4 weeks after cell transplantation (Figure 3, A, B). A scar area of $36.5 \% \pm 8.2 \%$ was detected in control animals 4 weeks after medium injection. Masson's Trichrome staining from animals of the skeletal myoblast and the stem cell group revealed a significant reduction of myocardial scar to $22.3 \% \pm 9.1 \%$ and
$19.8 \% \pm 7.6 \%$, respectively. This reduction was more prominent in animals that received transplantation of both cell types $(13.2 \% \pm 5.8 \%, P=.036$ and $P=.044$, compared with skeletal myoblasts and $\mathrm{AC}-133+$ cells, respectively) (Figure 3,C).

Double-cell Therapy With Skeletal Myoblasts and AC-133+ Cells Attenuates Apoptosis in the Border Zone and Distal Area

To evaluate the effect of cell-therapy types on surviving myocardium, we performed a TUNEL staining of both the border zone and the distal area and calculated the apoptotic index (Figure 4, A, B). Approximately two thirds of surviving cardiomyocytes were apoptotic in the peri-infarct area of control animals, whereas the extent of apoptosis was significantly lower after single-cell transplantation of skeletal myoblasts or AC-133+ cells (online Figures E3 and E4). Equivalent sections from the combination group revealed an even more profound attenuation of myocardial apoptosis in the border zone (Figure 4, $C$ ). Similar results were obtained after analysis of apoptotic rates in the remote area, indicating that the beneficial 

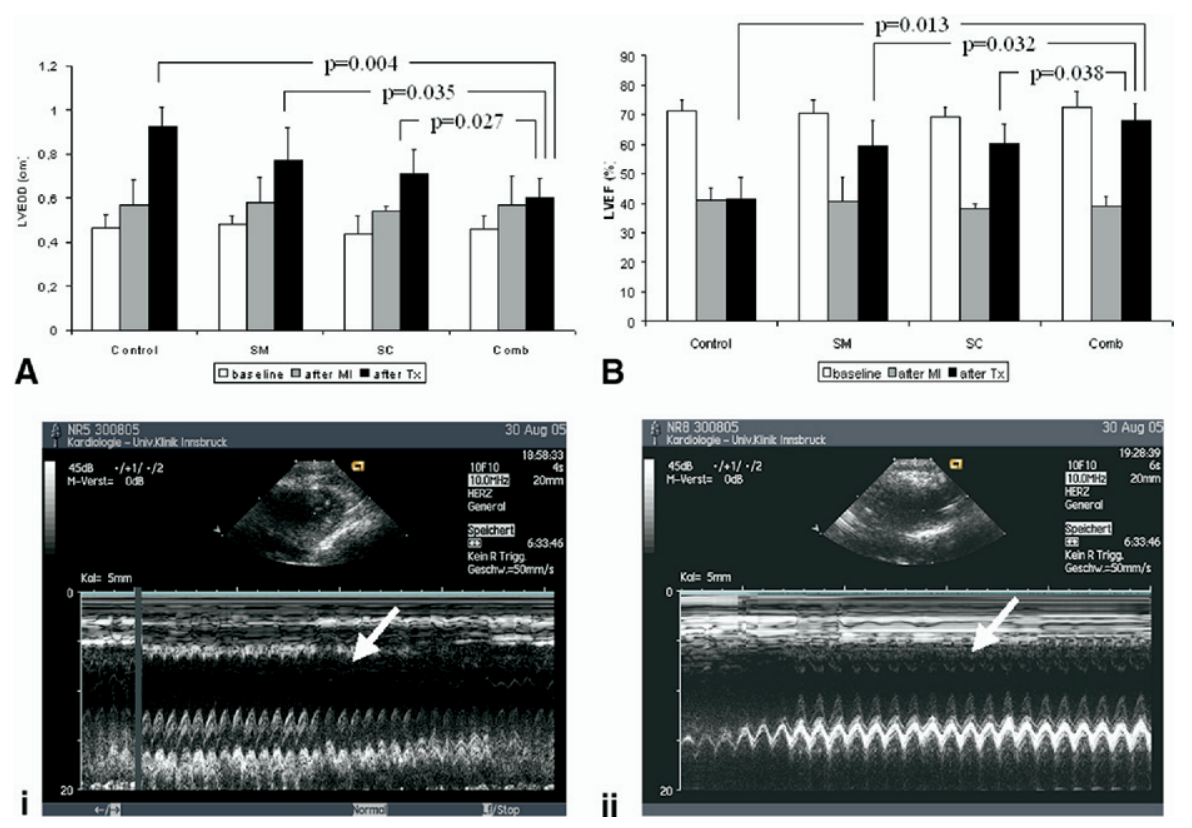

Figure 2. Combined transplantation of skeletal myoblasts and AC-133+ progenitors reverses left ventricular dilatation (A) and improves left ventricular function (B), as assessed by transthoracic echocardiography 4 weeks after cell transplantation (results expressed as mean \pm standard deviation). M-Mode echocardiography, i., 2 days after myocardial infarction; ii., 4 weeks after double-cell therapy. Animals received Roswell Park Memorial Institute injections in the scar and peri-infarct rim (control), homologous skeletal myoblasts in the scar (SM), human-derived AC-133+ cells in the peri-infarct rim (SC), homologous skeletal myoblasts and humanderived AC-133+ cells (Comb) in the scar and peri-infarct rim, respectively. Note anterior wall hypokinesia and left ventricular dilatation after myocardial infarction (arrow). Both findings are partially reversed 4 weeks after combined cell therapy (arrow). effect of double-cell therapy is not limited to the immediate ischemic tissue (Figure 4,D). To investigate the effect of combined cell therapy on the newly formed myotubes, we assessed apoptotic rates within engrafted skeletal myoblasts in the infarct scar. The apoptotic index was significantly lower in animals that received double-cell therapy $(0.42 \pm 0.19)$ compared with animals that received homologous skeletal myoblasts alone $(0.79 \pm$ $0.15, P=.0004)$.
Combined Transplantation of Skeletal Myoblasts and Angiopoietic Progenitors Increases Capillary Density in Chronic Ischemic Myocardium

Because both cells have been shown to increase neoangiogenesis in the ischemic heart, we next studied the effects of combined cell transplantation on capillary density in chronic ischemic myocardium (Figure 5, A, B). Capillary density in the border zone and scar area was significantly higher in rats after
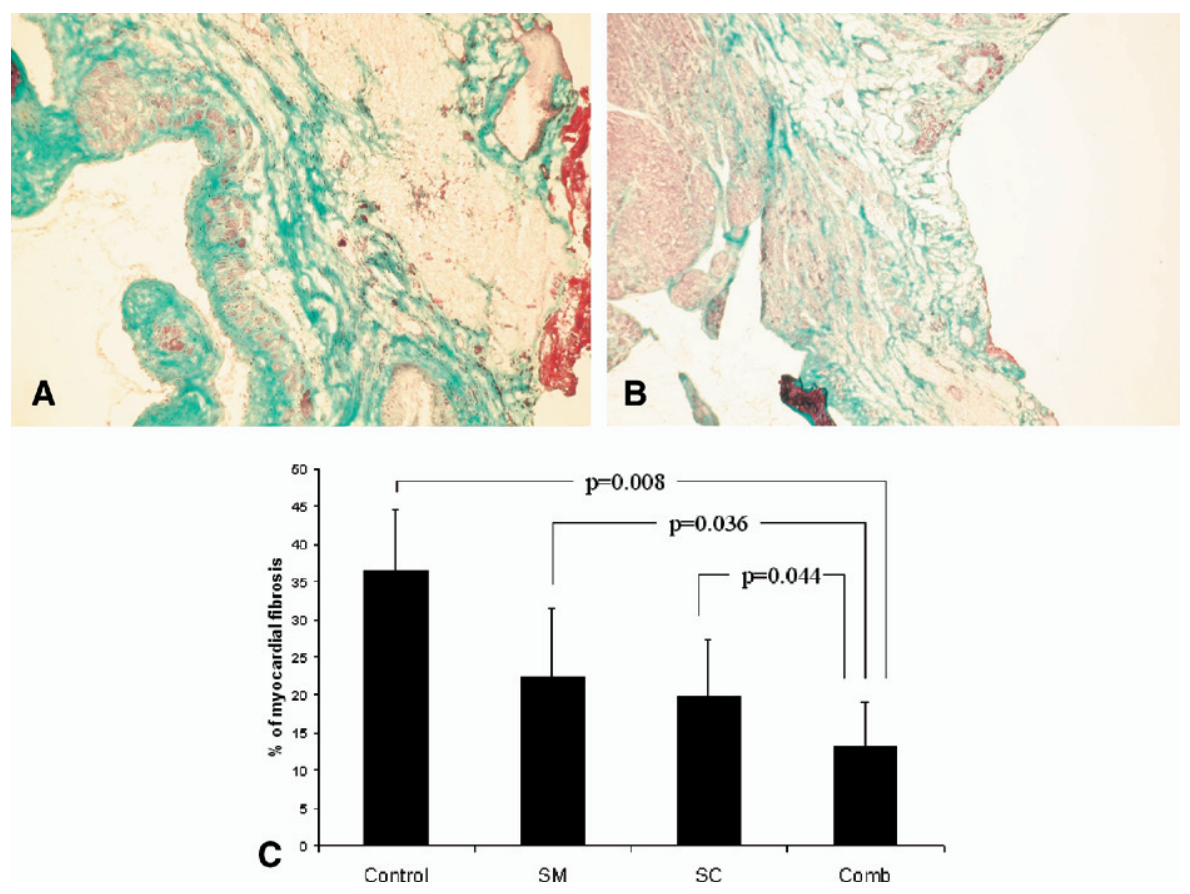

Figure 3. Combined transplantation of skeletal myoblasts and angiopoietic progenitors prevents myocardial fibrosis and results in reduced myocardial scar, as assessed by Masson's Trichrome staining 8 weeks after myocardial infarction. A, Scar area of a control animal 4 weeks after injection of culture medium (40× magnification). $B$, Scar area of an animal after myocardial infarction and 4 weeks after skeletal myoblasts transplantation $(40 \times$ magnification). C, Quantification of myocardial fibrosis 4 weeks after injection of culture medium (control), homologous skeletal myoblasts (SM), human-derived AC-133+ cells (SC), or combination of homologous skeletal myoblasts and human-derived AC-133+ cells. 

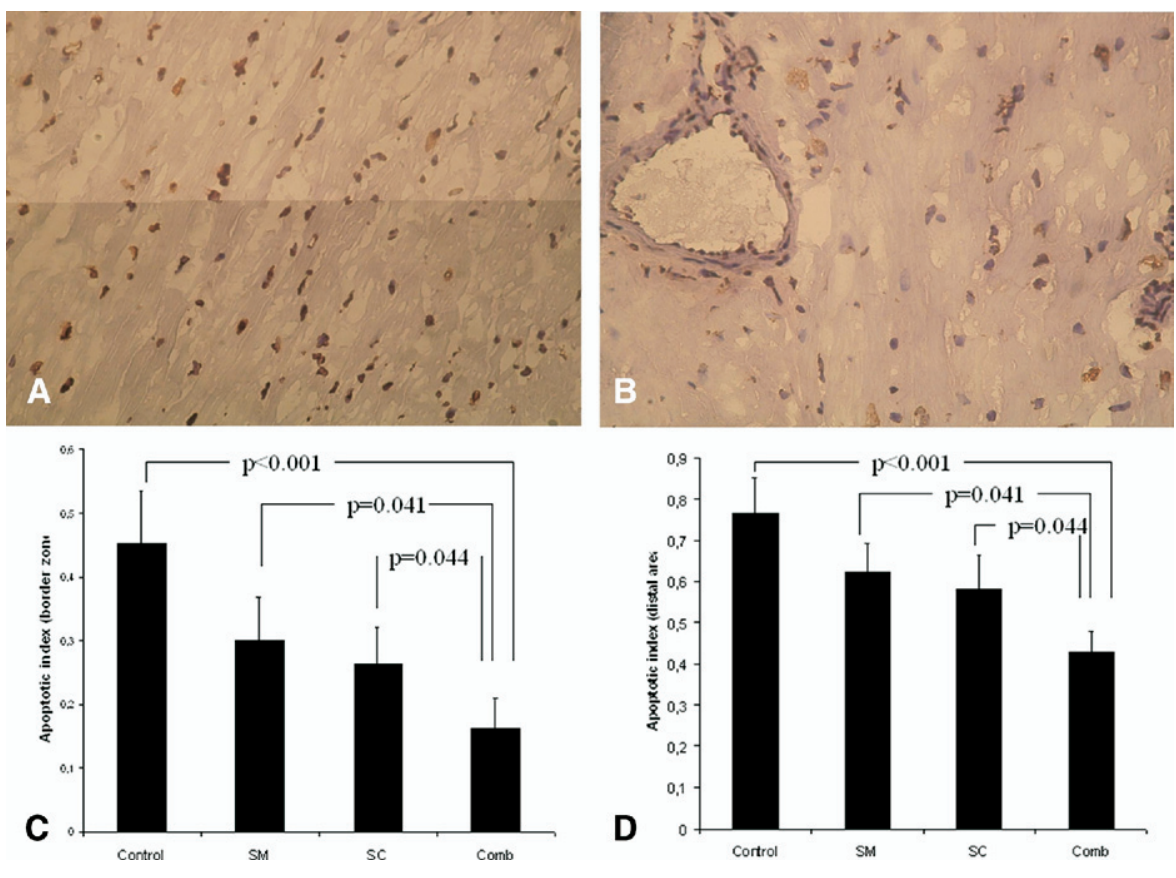

Figure 4. Double-cell therapy with skeletal myoblasts and AC-133+ cells attenuates apoptosis in the border zone and distal area, as assessed by TUNEL staining. TUNEL staining of the border zone 4 weeks after injections of (A) culture medium and (B) combination of homologous skeletal myoblasts and human-derived AC-133+ cells (brown and blue nuclei indicating apoptotic and non-apoptotic cells, respectively). Quantification of apoptotic index, calculated as the ratio of apoptotic cardiomyocytes in the total myocardial cell population in the border zone (C) and distal area (D). Results are expressed as mean \pm standard deviation. cell monotherapy compared with controls, with a trend of more capillaries in the group receiving AC-133+ cells than the group receiving skeletal myoblasts (online Figures E5 and E6). Combined transplantation of skeletal myoblasts and AC-133+ cells resulted in a 6.5- and 1.6-fold increase of capillary density compared with controls and skeletal myoblast monotherapy, respectively (Figure 5,C). Similar results were observed with regard to the capillary density in the infarct area (Figure $5, D$ ).

\section{Discussion}

In the current study we evaluated the effect of combined transplantation of skeletal myoblasts in the infarct area and angiopoietic progenitor cells in the border zone in a rodent model of ischemic heart failure. Although this approach has been described, ${ }^{14,15}$ this is the first study to introduce the concept of double-cell therapy in an environment of chronic myocardial ischemia. Other approaches included injections

\section{A}

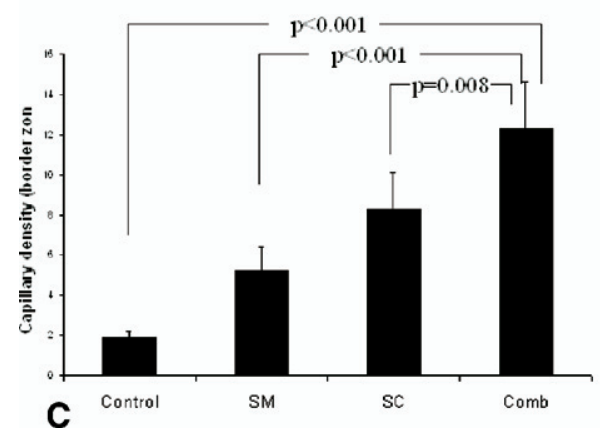

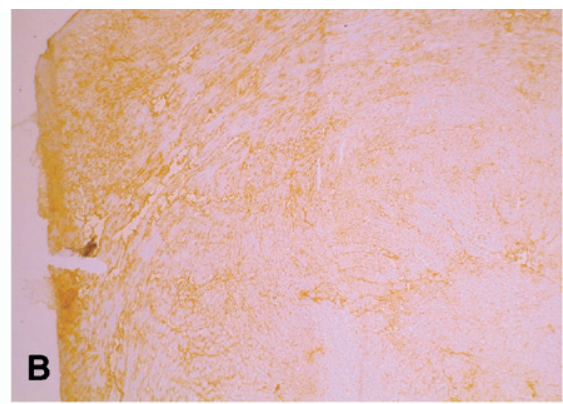

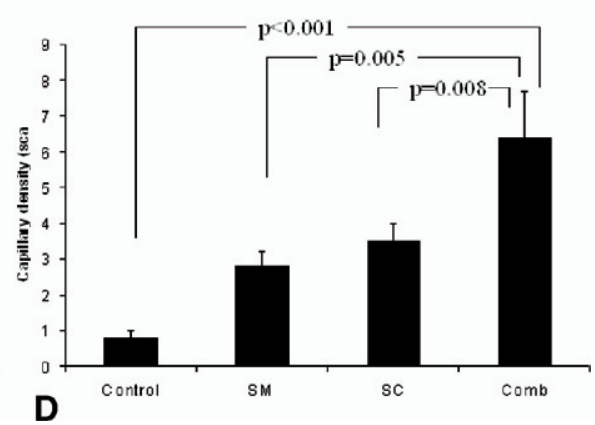

Figure 5. Degree of neovascularization in the peri-infarct area of chronic infarcted myocardium after injection of (A) culture medium and (B) combination of skeletal myoblasts and AC-133+ cells. Quantification of capillary density in the border zone (C) and scar (D) 4 weeks after cell transplantation. 
of modulated skeletal myoblasts with genetically enhanced growth factor secretion. ${ }^{16}$ In a previous study we hypothesized that skeletal myoblasts may attenuate the effect of chronic myocardial ischemia, provided that blood and oxygen supply from the peri-infarct area are not restricted. ${ }^{14}$ In the present study we selected a specific subgroup of endothelial progenitor cells that represent only $1 \%$ of the cells in whole bone marrow aspirates or whole blood from granulocyte colony-stimulating factor-mobilized patients ${ }^{17}$ and that have been shown to actively participate in new vessel formation. ${ }^{18}$ In this context, we injected AC-133+ angiopoietic progenitors in the peri-infarct area to increase neovascularization and to subsequently improve blood and oxygen supply in the ischemic myocardium.

We used in vivo and ex vivo studies to show the functional and morphologic effects of combined cell transplantation. Echocardiography studies revealed an impressive recovery of approximately $30 \%$ in LVEF in the combination group. This improvement was significantly higher than that reported by other authors, ${ }^{14,15}$ even 4 weeks post-myocardial infarction, after establishment of chronic heart failure and completion of the remodeling process. In this setting, myocardial apoptotic rates have been shown to be more than 3 times higher compared with those after acute myocardial infarction, whereas the regeneration capacity of native myocardium remains extremely low. ${ }^{19}$ Left ventricular dilatation after myocardial infarction, being the main remodeling parameter, was significantly reduced by using this therapeutic concept. This finding was also described in a model of semi-chronic ischemia, in which cell injections were performed 2 weeks after myocardial infarction and actually before completion of the remodeling process. ${ }^{15}$

Functional improvement correlated to histologic findings of myocardial fibrosis and apoptotic rates in the peri-infarct region and distal area. As proposed by other investigators, skeletal myoblasts can partially replace the myocardial scar, provided that they survive in sufficient numbers in the host. ${ }^{5}$ Whether the reduction of myocardial scar detected in animals that received AC-133+ cells is attributed to the formation of new cardiomyocytes, fusions, or limited scar expansion during remodeling remains to be investigated. Lower apoptotic rates in the peri-infarct region in stem cell-treated animals, in combination with the fact that no new myocytes were detected in the middle of the scar (data not shown), provide evidence that factors other than direct myocardial regeneration are involved.

The major finding of the study was that injections of angiopoietic progenitors in the border zone and skeletal myoblasts in the infarct scar resulted in a significantly higher neovascularization of the chronic ischemic myocardium. Several studies have underlined the angiogenic effect of AC-133+ cells, which is mainly attributed to expression of growth factors, such as basic fibroblast growth factor,
VEGF, and angiopoietin-1. ${ }^{12,20}$ Secretion of those factors has been shown to regulate the differentiation of mononuclear cells into endothelial cell lineage. ${ }^{21}$ Skeletal myoblasts, although lineage restricted, also demonstrate a significant angiogenic potential, which has been mainly attributed to only partially investigated paracrine properties $^{22}$ and to increased production of VEGF. ${ }^{6}$ This hypothesis was also verified by our findings, because increased vascularization was detected not only in the infarct scar, where myoblasts were actually injected, but also in the peri-infarct region.

Angiopoietic progenitor cells were detected in the area of injection (border zone) and the infarct scar. This demonstrates that injected cells not only survive in the ischemic heart but also migrate into the infarct area. Accordingly, other investigators were able to detect angiopoietic progenitors 4 weeks after cell transplantation in the scar with polymerase chain reaction, but not with fluorescence microscopy. ${ }^{23}$ We believe these differences can be attributed to the different protocols used or to fluorescence weakening caused by fusions between engrafted AC-133+ cells and the few residual in-scar cardiomyocytes. ${ }^{24}$ The higher vascular density detected in the infarct area of animals subjected to double-cell therapy, compared with those receiving AC-133+ cells alone, indicates the presence of an additional mechanism of neoangiogenesis. Inflammation mediators, such as tumor necrosis factor- $\alpha$, interleukin- 8 , or stromal cell-derived factor 1 , are less likely to stem from ischemic myocardium, because cytokine production and expression of chemoattractants decline within days after myocardial infarction. ${ }^{25}$ Molecular signals are not expected to be produced within the infarct area, because the number of residual cells remains low 4 weeks after infarction, and those cells express high apoptotic rates and subsequently cannot be functional. In this context it is unknown whether chemoattractants secreted from engrafted myoblasts are potent enough to induce migration and homing of AC-133+ cells into the scar. ${ }^{26}$ The fact that injected angiopoietic progenitors were detected in the scar only in rats that received double-cell therapy provides us with evidence that an interaction between the 2 cell types is present that results in enhanced migration of angiopoietic progenitors into the scar. Both the origin of the chemoattracting mechanisms and the potential myoblast-derived intercellular signaling should be addressed in future investigations.

The third interesting finding was the effect of combined cell therapy on the apoptosis rate of skeletal myoblasts. Although survival studies of the engrafted cells were not the aim of this study, we hypothesized that the functional benefit of this therapeutic concept was mainly attributed to a higher oxygen supply for the transplanted myoblasts, which may have warranted functional improvement. Engrafted myotubes, detected more than 1 month after myoblast trans- 
plantation, presented high apoptotic rates, showing that although these cells are believed to be ischemia-resistant, they may possess a higher functionality potential, which is therefore limited mainly because of apoptotic mechanisms. Additional injections of angiopoietic progenitor cells in the border zone resulted in significantly decreased apoptotic rates of engrafted myotubes. This finding, in combination with the fact that vascular density was significantly increased in those animals, provides evidence that functional improvement after double-cell therapy is mainly attributed to the improvement of blood supply for the engrafted myoblasts in the hostile environment of the scar.

The complexity of the study setting and the high mortality rates of infectious prone nude rats with chronic heart failure did not allow a dose-escalation analysis to specify the ideal dose required or elaborated investigation of arrhythmias. For this reason, we injected commonly accepted numbers of skeletal myoblasts and angiopoietic progenitors. Another limitation of this study is the absence of survival analysis, because this could not performed in a reliable way in the current study setting. A direct comparison of both cell types in terms of functional and morphologic improvement in ischemic heart failure has been addressed by other investigators $^{23}$ and exceeded the purpose of this study.

We conclude that combined transplantation of skeletal myoblasts and AC-133+ angiopoietic progenitor cells improves heart function, partially reverses myocardial remodeling, and reduces apoptosis and scar formation in a model of chronic myocardial infarction. This functional and morphologic improvement is mainly associated with the increased neovascularization. Although both cell types seem to play different roles in cardiac regeneration and neoangiogenesis, the presence of cellular interactions at the molecular level may be responsible for the enhanced efficacy of combination therapy. However, the exact mechanism of action and the extent to which both cell types communicate with each other remain to be investigated. Testing of this concept in large animal models may be useful to address logistic issues such as harvesting of both homologous skeletal myoblasts and angiopoietic progenitors before organizing clinical trials.

\section{References}

1. Taylor DA, Atkins BZ, Hungspreugs P, et al. Regenerating functional myocardium: improved performance after skeletal myoblast transplantation. Nat Med. 1998;4:929-33.

2. Murry CE, Wiseman RW, Schwartz SM, Hauschka SD. Skeletal myoblast transplantation for repair of myocardial necrosis. $J$ Clin Invest. 1996;98:2512-23.

3. Menasché P, Hagège AA, Vilquin JT, et al. Autologous skeletal myoblast transplantation for severe post infarction left ventricular infarction. J Am Coll Cardiol. 2003;41:1078-83.

4. Dib N, Michler RE, Pagani FD, et al. Safety and feasibility of autologous myoblast transplantation in patients with ischemic cardiomyopathy: four-year follow-up. Circulation. 2005;112:1748-55.

5. Tambara K, Sakakibara Y, Sakaguchi G, et al. Transplanted skeletal myoblasts can fully replace the infarcted myocardium when they survive in the host in large numbers. Circulation. 2003;108(Suppl I): II259-63.

6. Yau TM, Li G, Weisel RD, et al. Vascular endothelial growth factor transgene expression in cell-transplanted hearts. J Thorac Cardiovasc Surg. 2004;127:1180-7.

7. Pagani FD, DerSimonian H, Zawadzka A, et al. Autologous skeletal myoblasts transplanted to ischemia-damaged myocardium in humans. Histological analysis of cell-survival and differentiation. $J$ Am Coll Cardiol. 2003;41:879-88.

8. Leobon B, Garcin I, Menasche P, Vilquin JT, Audinat E, Charpak S. Myoblasts transplanted into rat infarcted myocardium are functionally isolated from the host. Proc Natl Acad U S A. 2003;100:7808-11.

9. Reyes M, Dudek A, Jahagirdar B, Koodie L, Marker PH, Verfaillie $\mathrm{CM}$. Origin of endothelial progenitors in human postnatal bone marrow. J Clin Invest. 2002;109:337-46.

10. Kocher AA, Schuster MD, Szabolcs MJ, et al. Neovascularization if ischemic myocardium by human bone marrow-derived angioblasts prevents cardiomyocyte apoptosis, reduces remodeling and improves cardiac function. Nat Med. 2001;7:430-6.

11. Peichev M, Naiyer AJ, Pereira D, et al. Expression of VEGFR-2 and AC133 by circulating human $\mathrm{CD} 34(+)$ cells identifies a population of functional endothelial precursors. Blood. 2000;95:952-8.

12. Kucia M, Dawn B, Hunt G, et al. Cell expressing early cardiac markers reside in the bone marrow and are mobilized into the peripheral blood after myocardial infarction. Circ Res. 2004;95:1191-9.

13. Murry CE, Soonpaa MH, Reinecke H, et al. Hematopoietic stem cells do not transdifferentiate into cardiac myocytes in myocardial infarcts. Nature. 2004;428:664-8.

14. Ott HC, Bonaros N, Marksteiner R, et al. Combined transplantation of skeletal myoblasts and bone marrow stem cells for myocardial repair in rats. Eur J Cardiothorac Surg. 2004;25:627-34.

15. Memon IA, Sawa Y, Miyagawa S, Taketani S, Matsuda H. Combined autologous cellular cardiomyoplasty with skeletal myoblasts and bone marrow cells in canine heart for ischemic cardiomyopathy. $J$ Thorac Cardiovasc Surg. 2005;130:646-53.

16. Tambara K, Premaratne GU, Sakaguchi G, et al. Administration of control-released hepatocyte growth factor enhances the efficacy of skeletal myoblast transplantation in rat infarcted heart by greatly increasing both quantity and quality of the graft. Circulation. 2005; 112(9 Suppl):I129-34.

17. Salven P, Mustjoki S, Alitalo R, Alitalo K, Rafii S. VEGFR-3 and CD133 identify a population of CD34+ lymphatic/vascular endothelial precursor cells. Blood. 2003;101:168-72.

18. Gehling U, Ergün S, Schumacher U, et al. In vitro differentiation of endothelial cells from AC133-positive progenitor cells. Blood. 2000; 95:3106-12.

19. Urbanek K, Torella D, Sheikh F, et al. Myocardial regeneration by activation of multipotent cardiac stem cells in ischemic heart failure. Proc Natl Acad Sci U S A. 2005;102:8692-7.

20. Galli D, Innocenzi A, Staszewsky L, et al. Mesangioblasts, vessel-associated multipotent stem cells, repair the infarcted heart by multiple cellular mechanisms: a comparison with bone marrow progenitors, fibroblasts, and endothelial cells. Arterioscler Thromb Vasc Biol. 2005;25:692-7.

21. Shi Q, Rafii S, Wu NH, et al. Evidence of circulating bone marrowderived endothelial cells. Blood. 1998;92:362-7.

22. Murtuza B, Suzuki K, Bou-Gharios G, et al. Transplantation of skeletal myoblasts secreting an IL-1 inhibitor modulates adverse remodeling in infarcted murine myocardium. Proc Natl Acad Sci U S A. 2004;101:4216-21.

23. Agbulut $\mathrm{O}$, Vandervelde $\mathrm{S}, \mathrm{Al}$ Attar N, et al. Comparison of human skeletal myoblasts and bone marrow-derived CD 133+ progenitors for repair of infarcted myocardium. $J$ Am Coll Cardiol. 2004;44:458-63.

24. Nygren JM, Jovinge S, Breitbach M, et al. Bone marrow-derived hematopoietic cells generate cardiomyocytes at a low frequency through cell fusion, but not transdifferentiation. Nat Med. 2004;10:494-501.

25. Kacimi R, Karliner JS, Koudssi F, Long CS. Expression and regulation of adhesion molecules in cardiac cells by cytokines: response to hypoxia. Circ Res. 1998;82:576-86.

26. Kocher AA, Schuster MD, Bonaros N, et al. Myocardial homing and neovascularization by human bone marrow angioblasts is regulated by IL-8/Gro CXC chemokines. J Moll Cell Cardiol. 2006;40:455-64. Epub 2006 Jan 24. 

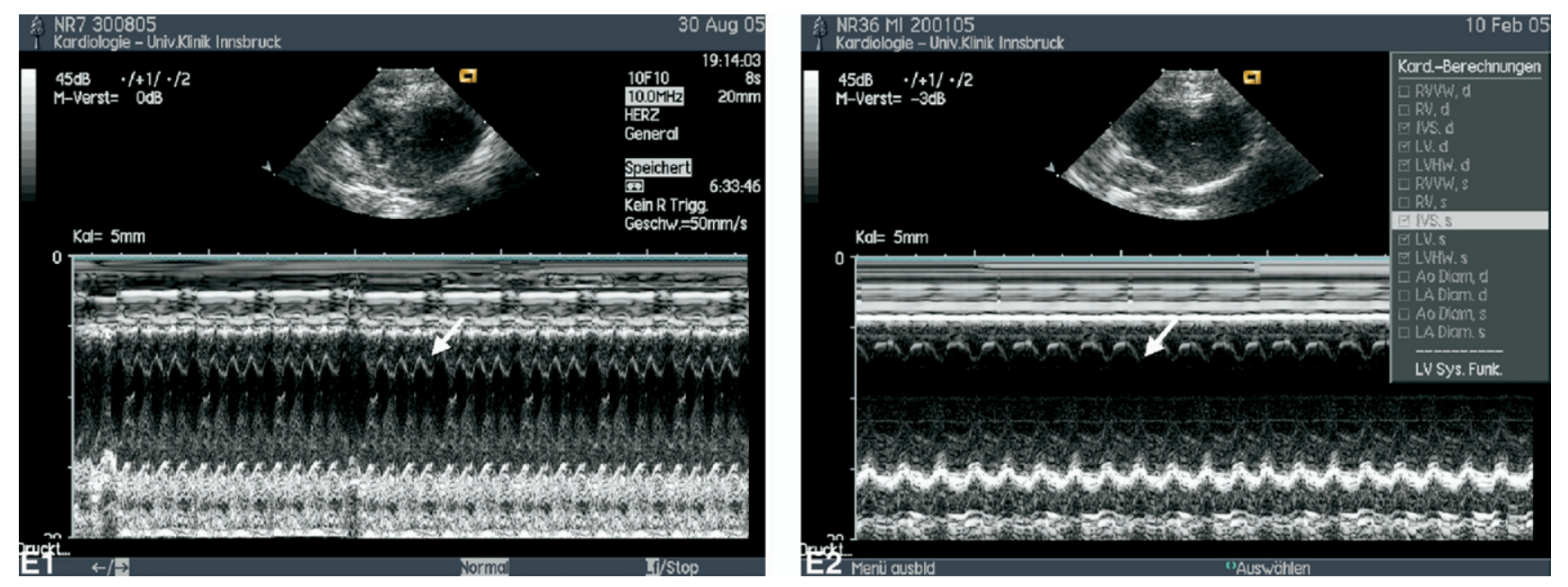

Figures E1 and E2. M-Mode echocardiography before myocardial infarction (E1) and 4 weeks after cell monotherapy (E2). Note anterior wall movement and normal dimensions of the left ventricle before myocardial infarction (arrow, Figure E1) and 4 weeks after cell monotherapy (arrow, Figure E2).
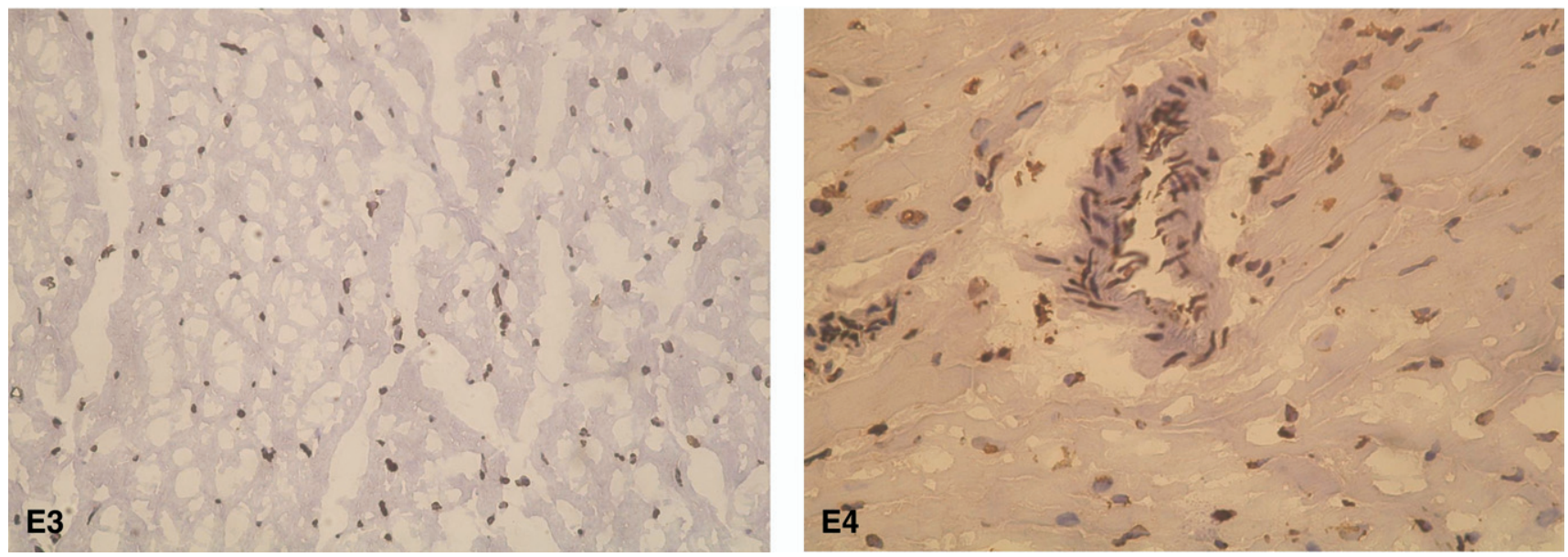

Figures E3 and E4. Double-cell therapy with skeletal myoblasts and AC-133+ cells attenuates apoptosis in the border zone and distal area, as assessed by TUNEL staining. TUNEL staining of the border zone 4 weeks after injections of homologous skeletal myoblasts (E3) and human-derived AC-133+ cells (E4) (brown and blue nuclei indicating apoptotic and non-apoptotic cells, respectively). 

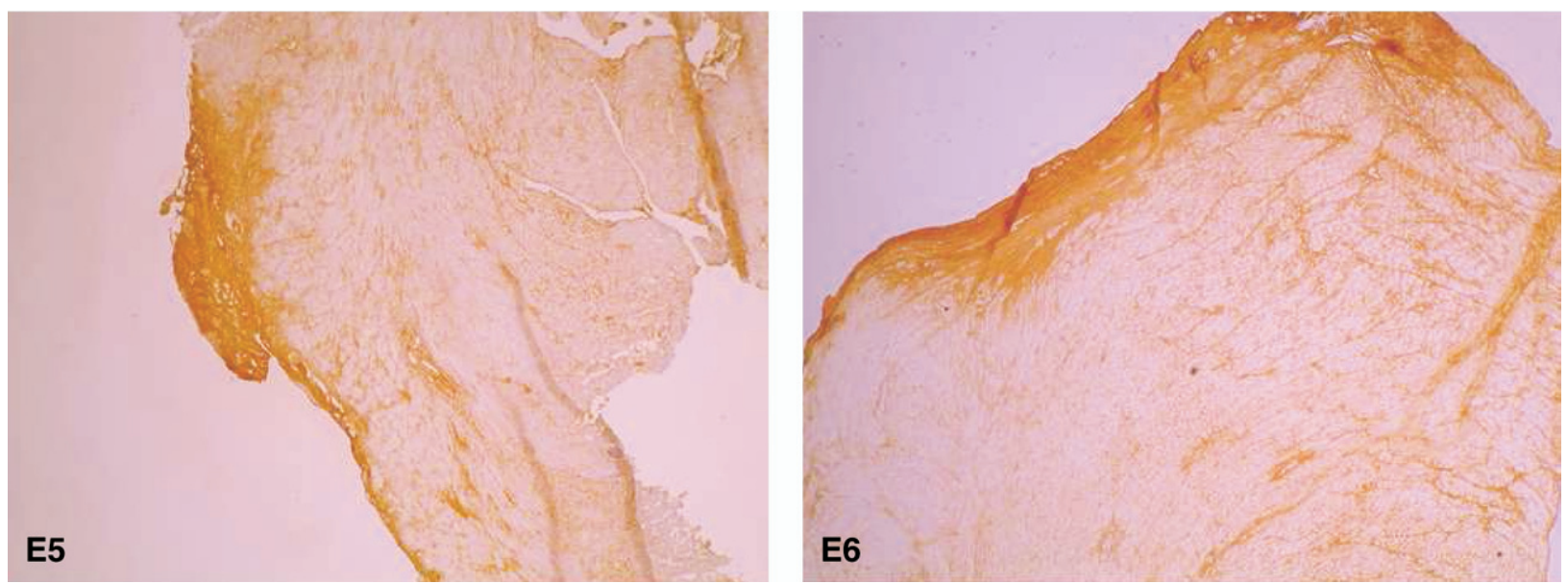

Figures E5 and E6. Degree of neovascularization in the peri-infarct area of chronic infarcted myocardium after injection of skeletal myoblasts (E5) and AC-133+ cells (E6) as assessed by means of CD34 staining of endothelial cells. 